\title{
Avoidance of tolerance and lack of rebound with intermittent dose titrated transdermal glyceryl trinitrate
}

K M Fox, H J Dargie, J Deanfield, A Maseri, on behalf of the Transdermal Nitrate Investigators

Royal Brompton National Heart and Lung Hospital, London K M Fox

Western Infirmary, Glasgow

H J Dargie

Hospital for Sick Children, London J Deanfield

Hammersmith Hospital, London A Maseri

Transdermal Nitrate Investigators: Wexham Park Hospital, Slough, Berkshire

R A Blackwood

Royal Gwent Hospital, Newport, Gwent

J Davies

C James

Wycombe General Hospital, High Wycombe,

Buckinghamshire

W G Hendry

Royal Infirmary,

Glasgow

I Hutton

East Birmingham Hospital, Birmingham R G Murray M Salih

Northern General Hospital, Sheffield G D Oakley

Taunton and Somerset Hospital, Taunton

J Sanderson

E Wood

Correspondence to Dr K M Fox, Royal Brompton National Heart and Lung Hospital, Sydney Street, London SW3 6NP.

Accepted for publication 6 February 1991
Abstract

Objectives-To investigate the efficacy of transdermal glyceryl trinitrate given continuously and with a nocturnal nitrate free period.

Design-Double blind placebo controlled study with two parallel limbs.

Setting-Multicentre trial.

Patients-52 patients randomised to receive either continuous treatment ( 23 patients) or intermittent treatment with an individually titrated dose (29 patients) for 14 days: both treatments were compared with placebo in a crossover fashion.

Intervention-Continuous treatment with $10 \mathrm{mg}$ per 24 hours of transdermal glyceryl trinitrate or intermittent transdermal glyceryl trinitrate titrated to give an arbitrary $10 \mathrm{~mm} \mathrm{Hg}$ drop in systolic blood pressure (mean dose $18.2 \mathrm{mg}$ ) given over approximately 16 hours.

Main outcome measure-Treadmill exercise stress testing and ambulatory monitoring of the ST segment after 14 days' treatment.

Results-After 14 days' intermittent treatment resting supine and standing systolic blood pressure fell by $7.5 \mathrm{~mm} \mathrm{Hg}$ (95\% confidence interval $2 \cdot 7$ to $12 \cdot 2)$ and $9.0 \mathrm{~mm} \mathrm{Hg}(95 \%$ CI 3.4 to 14.5$)$ respectively ( $p<0.01$ ); resting heart rate was unchanged. Mean heart rate at $1 \mathrm{~mm}$ ST segment depression rose by 11.9 beats/ min (CI 1.1 to 23.7$)(p<0.05)$, mean time to onset of angina increased by 59 seconds (CI 10.8 to 108) ( $<<0.05)$, and total exercise duration increased by 40 seconds $(p<0.05)$. These changes were not seen after continuous treatment. The frequency of ischaemic episodes was not reduced with either regimen nor was the circadian distribution of these episodes altered, in particular nocturnal episodes did not increase during intermittent treatment.

Conclusion-Tolerance to glyceryl trinitrate was avoided by the use of individually titrated doses administered with a nocturnal nitrate free period. There was no evidence of "rebound" on ambulatory monitoring during this treatment.
Organic nitrates have been used to treat angina pectoris for over a century ${ }^{1}$ but their short duration of action and rapid hepatic metabolism have limited their prophylactic use. Molecular manipulations and formulation developments have considerably extended the duration of action of these drugs and led to their more widespread use. Transdermal nitrate patches maintain constant plasma concentrations of glyceryl trinitrate over 24 hours but there is controversy about tolerance and whether it detracts from the role of nitrates in the prophylactic treatment of angina. ${ }^{2-4}$ The antianginal benefits of glyceryl trinitrate in the first few hours after the application of a patch are well recognised ${ }^{5}$ but several studies have found either a partial ${ }^{6}$ or complete loss of efficacy 24 hours after application of a patch. ${ }^{78}$ A nitrate free interval avoided the development of tolerance during long term treatment with isosorbide dinitrate, ${ }^{9}$ isosorbide mononitrate, and also with transdermal patches. ${ }^{10}$

The mechanisms of nitrate tolerance are not understood. It is thought that nitrate action is brought about by a cascade of events that affect the oxidation of important sulphydryl groups. ${ }^{11}$ These are necessary for the production of intermediary derivatives of S-nitrosothiol, which are required to produce cyclic guanosine monophosphate, the mediator for vascular smooth muscle relaxation. ${ }^{1213}$ Continued administration of nitrates induces tolerance by reducing the intracellular concentration of reduced sulphydryl groups to a critical concentration.

We compared the effects of the continuous 24 hour administration of glyceryl trinitrate with that of a titrated dose and a nocturnal nitrate free interval.

\section{Patients and methods}

Ninety seven patients ( 79 men and 18 women) aged 34-72 years (mean 56.6 years) attending cardiology outpatient clinics at seven participating centres throughout England, Scotland, and Wales were selected for screening according to the following criteria. All had a history of chronic stable angina pectoris on effort caused by ischaemic heart disease for a minimum of three months before their selection and a positive exercise stress test (with 
electrocardiographic changes). All patients were ambulant, able to maintain accurate diaries of anginal attacks, and were capable of being managed for the duration of the study without the use of $\beta$ blocking agents, calcium antagonists, or long acting oral nitrates. Patients were specifically excluded from study if they had had a documented myocardial infarction in the preceding three months; electrocardiographic abnormalities that might have interfered with the interpretation of the ST segment; a diastolic blood pressure $>100$ $\mathrm{mm} \mathrm{Hg}$; or significant renal, hepatic, haematological, or other clinically relevant concurrent conditions.

All eligible patients entered a one week, open run-in period and were maintained on sublingual glyceryl trinitrate alone. They performed a symptom limited treadmill exercise stress test according to a modified Bruce protocol. To be included in the treatment phases of the study patients had to show a positive response to sublingual glyceryl trinitrate during a second exercise stress test performed at least one hour and up to three hours later, 10 minutes after the patient had taken one $(500 \mu \mathrm{g})$ sublingual tablet of glyceryl trinitrate. The response was defined as positive if the exercise time needed to cause $1 \mathrm{~mm} \mathrm{ST}$ segment depression was $\geqslant 2$ minutes longer than during the first exercise test. During the second test, the workload was not increased beyond that required to produce $1 \mathrm{~mm}$ of ST segment depression in the first test; this avoided the false negative results caused by a higher workload.

The 85 patients who responded to sublingual glyceryl trinitrate were randomised to receive either a placebo controlled continuous or a placebo controlled intermittent treatment regimen lasting one month. After randomisation to the treatment regimens patients were re-randomised to active treatment followed by placebo treatment or vice versa in a balanced crossover design $(2 \times 2$ weeks $)$. During continuous treatment patients were treated with transdermal patches delivering $10 \mathrm{mg}$ glyceryl trinitrate over 24 hours (Ciba) or a matching placebo. We selected this regimen because this was the maximum recommended dose approved by the regulatory authorities. The regimen of intermittent treatment used a dose that was titrated to achieve a $10 \mathrm{~mm} \mathrm{Hg}$ fall in systolic blood pressure. Blood pressure was recorded two hours after the application of a patch. If a $10 \mathrm{~mm} \mathrm{Hg}$ fall was not seen, another $10 \mathrm{mg} / 24 \mathrm{~h}$ patch was applied and observation repeated to a maximum of four patches $(40 \mathrm{mg} / 24 \mathrm{~h})$. Patients were maintained at their titrated dose for the duration of the study.

\section{EXERCISE STRESS TEST}

Patients exercised on a treadmill according to a modified Bruce protocol in the morning, three to five hours after a patch was applied. The patient was tested at the same time of day at the end of each two week treatment period. ST segment depression was measured $80 \mathrm{~ms}$ after the $J$ point in the precordial lead that had first shown $1 \mathrm{~mm}$ ST segment depression during the first test. Significant ST segment depression was defined as planar or downsloping ST segment depression of $\geqslant 1 \mathrm{~mm}$ measured $0.08 \mathrm{~s}$ after the $\mathrm{J}$ point. The following indices were recorded: time to $1 \mathrm{~mm} \mathrm{ST}$ segment depression, heart rate at $1 \mathrm{~mm} \mathrm{ST}$ segment depression, maximum ST segment depression, time to onset of angina, and total exercise time. All the electrocardiograms were examined and reported in a blind manner by one analyst.

\section{HOLTER MONITORING}

Holter monitoring was performed at the end of each treatment period. All patients underwent 48 hours of ambulatory ST segment monitoring with pre-gelled electrodes to record two bipolar leads: one an anterior lead CM5 and the other an inferior lead. The sites and method of application of these electrodes have been described elsewhere. ${ }^{14}$ Twochannel recordings were then obtained on magnetic tape by a frequency modulated dual channel recorder (Oxford Medilog 2, frequency response $0 \cdot 05-40 \mathrm{~Hz}$ ). The frequency response of this system is sufficient to record and display the ST segment accurately. The tapes were then visually analysed at 60 times normal speed by an Oxford Medilog MA20 scanner; all printouts were recorded at 25 $\mathrm{mm} / \mathrm{s}$. Significant ST segment depression was defined as planar or downsloping ST segment shift of $\geqslant 1 \mathrm{~mm}$ measured $0.08 \mathrm{~s}$ after the J point that persisted for $>30 \mathrm{~s}$. Significant ST segment elevation was defined as an upward shift of the ST segment of $\geqslant 1 \mathrm{~mm}$ at the J point compared with the resting recording. Changes in the $T$ wave vector were not regarded as evidence of myocardial ischaemia unless they were accompanied by significant ST segment changes.

\section{DIARY CARD}

Diary cards were supplied to record angina episodes during a two week period. The weekly attack rate was calculated by summing the daily events occurring over two weeks and standardised to obtain a mean weekly figure. Glyceryl trinitrate tablet consumption was recorded and analysed in a similar fashion.

\section{STATISTICAL ANALYSIS}

Statistical analysis was based on the mean (or median) values calculated at each time point of the study: initial visit, end of run-in, end of period 1 , and end of period 2 . The haemodynamic indices of the two treatment sequence groups were compared at baseline (end of run-in) by Student's $t$ test. The effects of active and placebo treatment during the study were compared by the method of crossover analysis. Koch's non-parametric crossover method was used to compare the two treatment effects on the variables in the exercise stress test and on the angina diary cards.

Ambulatory monitoring results are expressed as a mean and standard deviation per 24 hours. Discrete data were analysed by the 
Patients screened

Negative screening exercise stress test

Positive screening exercise stress test

Negative nitrate response test

Positive nitrate response test Randomised to continuous treatment

Randomised to intermittent treatment

Protocol violations / ineligible

Treatment withdrawals

Completed the study
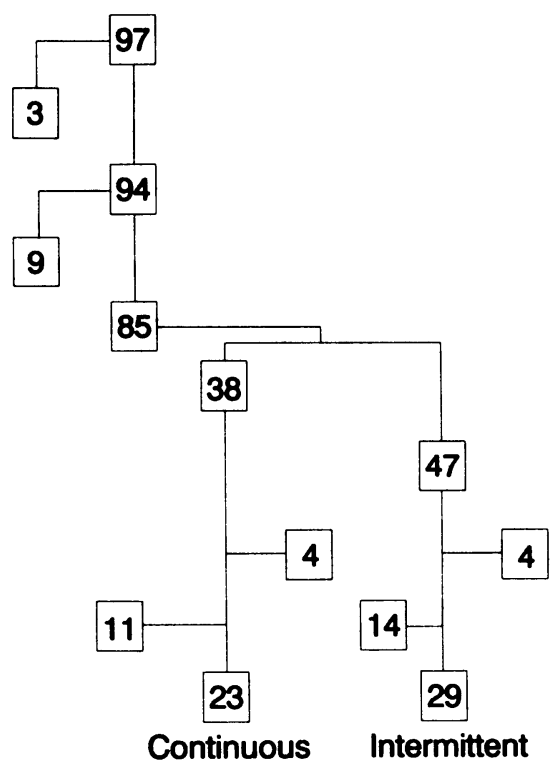

Figure 1 Flow chart to show the selection of patients to the study.

Wilcoxon signed rank test for paired observations during the double blind phase of the study. Fourier analysis was used on the hourly frequencies and durations of total ischaemia. The residual squares error was used to determine the optimum number of fitted harmonics, which was found to be a two harmonic fit, and the correlation coefficient of the fitted curve. The day was divided into four six-hour periods (01.30-07.30, 07.30-13.30, 13.3019.30 , and 19.30-01.30), and a $\chi^{2}$ test with Yates's correction for continuity was applied to determine whether there was a significant excess of episodes in the morning (07.3013.30) compared with the mean of the other three periods.

Ethical approval was obtained from each of the hospital medical committees.

\section{Results}

Ninety seven patients were screened. There were 85 nitrate responders: 38 were allocated to continuous treatment and 47 to intermittent treatment (fig 1). Twenty three patients completed the continuous treatment study and 29 patients completed the intermittent treatment study. For the intermittent treatment the mean individually titrated dose needed to cause a 10 $\mathrm{mm} \mathrm{Hg}$ drop in resting systolic blood pressure was $18 \mathrm{mg}$ given over $12 \mathrm{~h} ; 11$ patients required one patch, 14 patients two patches, two patients three patches, and two patients four patches.
The demographic indices of the treatment groups were well matched.

\section{HAEMODYNAMIC EFFECTS}

After two weeks of continuous $24 \mathrm{~h}$ treatment with one $10 \mathrm{mg} / \mathrm{h}$ patch applied daily no clinically or statistically significant differences were found compared with placebo treatment for the following variables: heart rate and systolic and diastolic blood pressures when supine and standing. Similarly no significant differences were seen in the mean resting heart rate or supine and standing diastolic blood pressures compared with placebo treatment after two weeks' active treatment with individually titrated doses of transdermal glyceryl trinitrate. With intermittent treatment, however, the resting systolic standing and supine blood pressures were significantly reduced $(\mathrm{p}<0.01)$ (table 1$)$.

\section{EXERCISE TESTING}

No significant differences in favour of transdermal glyceryl trinitrate were found after continuous application compared with placebo for any of the measured exercise test end points. In contrast, intermittent treatment significantly increased the mean total exercise by 36 seconds $(p<0.05)$, the mean time to the onset of angina by 46 seconds ( $p<0.05$ ), and the heart rate at $1 \mathrm{~mm}$ of ST segment depression was increased by 9 beats/min $(p<0.05)$. In addition, time to $1 \mathrm{~mm}$ ST segment depression increased by 49 seconds and maximum ST segment depression was reduced by $0.11 \mathrm{~mm}$; though these findings show a positive trend, they were not statistically significant (table 2 ).

\section{HOLTER MONITORING}

Treatment with continuous and transdermal glyceryl trinitrate did not affect the frequency of either the silent episodes of ST segment depression per $24 \mathrm{~h}$ or the total number of episodes of ST segment depression per $24 \mathrm{~h}$. Similarly, neither the duration nor the severity of ST segment changes was affected by either treatment.

Figures 2 and 3 show the circadian distribution of the episodes of total ischaemia recorded during the placebo phase and during the two treatments. A similar circadian pattern was seen during the two treatment periods and the placebo period, with a primary peak in the morning hours and a smaller secondary peak in the evening; very few episodes were recorded during the night before 07.00 during any of the treatment periods.

Table 1 Haemodynamic results (mean) of continuous and intermittent dosing with glyceryl trinitrate

\begin{tabular}{|c|c|c|c|c|c|c|c|}
\hline \multirow[b]{2}{*}{ Variable } & \multicolumn{3}{|c|}{ Continuous dosing } & \multicolumn{3}{|c|}{ Intermittent dosing } & \multirow{2}{*}{$\begin{array}{l}\text { Confidence } \\
\text { interval }\end{array}$} \\
\hline & Active & Placebo & p Value & Active & Placebo & p Value & \\
\hline $\begin{array}{l}\text { Heart rate (beats } / \mathrm{min}) \\
\text { Supine systolic } \mathrm{BP}(\mathrm{mm} \mathrm{Hg}) \\
\text { Supine diastolic } \mathrm{BP}(\mathrm{mm} \mathrm{Hg}) \\
\text { Standing systolic BP }(\mathrm{mm} \mathrm{Hg}) \\
\text { Standing diastolic BP }(\mathrm{mm} \mathrm{Hg})\end{array}$ & $\begin{array}{r}74 \cdot 5 \\
142 \cdot 6 \\
86 \cdot 3 \\
138 \cdot 8 \\
86 \cdot 7\end{array}$ & $\begin{array}{r}75 \cdot 1 \\
133 \cdot 8 \\
86 \cdot 3 \\
135 \cdot 4 \\
86 \cdot 8\end{array}$ & $\begin{array}{l}\text { NS } \\
\text { NS } \\
\text { NS } \\
\text { NS } \\
\text { NS }\end{array}$ & $\begin{array}{r}76 \cdot 0 \\
130 \cdot 8 \\
83 \cdot 3 \\
126 \cdot 8 \\
84 \cdot 0\end{array}$ & $\begin{array}{r}78 \cdot 0 \\
137 \cdot 4 \\
86 \cdot 2 \\
135 \cdot 8 \\
85 \cdot 1\end{array}$ & $\begin{array}{l}\text { NS } \\
<0.01 \\
\text { NS } \\
<0.01 \\
\text { NS }\end{array}$ & $\begin{array}{l}2 \cdot 7-12 \cdot 2 \\
3 \cdot 4-14 \cdot 5\end{array}$ \\
\hline
\end{tabular}

BP, blood pressure. NS, not statistically significant at $\mathrm{p}<0.05$ level. 
Table 2 Results (mean) of exercise testing during continuous dosing and intermittent dosing with glyceryl trinitrate

\begin{tabular}{|c|c|c|c|c|c|c|}
\hline \multirow[b]{2}{*}{ Variable } & \multicolumn{3}{|c|}{ Continuous dosing } & \multicolumn{3}{|c|}{ Intermittent dosing } \\
\hline & Active & Placebo & p Value & Active & Placebo & p Value \\
\hline $\begin{array}{l}\text { Maximum exercise duration (min) } \\
\text { Time to } 1 \mathrm{~mm} \text { ST depression (min) } \\
\text { HR at } 1 \mathrm{~mm} \text { ST depression (beats/min) } \\
\text { Maximum ST depression (min) } \\
\text { Onset of angina (min) }\end{array}$ & $\begin{array}{r}10 \cdot 9 \\
8 \cdot 3 \\
115 \cdot 4 \\
1.8 \\
7 \cdot 4\end{array}$ & $\begin{array}{r}11.5 \\
9 \cdot 3 \\
119 \cdot 0 \\
1 \cdot 6 \\
8 \cdot 8\end{array}$ & $\begin{array}{l}\text { NS } \\
\text { NS } \\
\text { NS } \\
\text { NS } \\
\text { NS }\end{array}$ & $\begin{array}{r}11 \cdot 3 \\
8 \cdot 7 \\
120 \cdot 5 \\
1 \cdot 3 \\
9 \cdot 4\end{array}$ & $\begin{array}{r}10.7 \\
7.9 \\
111.9 \\
1.4 \\
8.6\end{array}$ & $\begin{array}{l}<0.05 \\
\text { NS } \\
<0.05 \\
\text { NS } \\
<0.05\end{array}$ \\
\hline
\end{tabular}

NS, not statistically significant at $\mathrm{p}<0.05$ level.

ANGINA ATTACK RATES

The incidence of angina during treatment with placebo was low (6.6 attacks/week). Neither active treatment significantly changed this rate. The consumption of glyceryl trinitrate tablets was similarly unaltered. The incidence of nocturnal episodes was similar in all active and placebo treatment phases.

WITHDRAWALS FROM TREATMENT

Of the 85 patients who were randomised to treatment, 25 patients were withdrawn from the study, 11 receiving continuous treatment and 14 receiving intermittent treatment. A total of $17(20 \%)$ patients were withdrawn because of headache (14 active, three placebo), and four
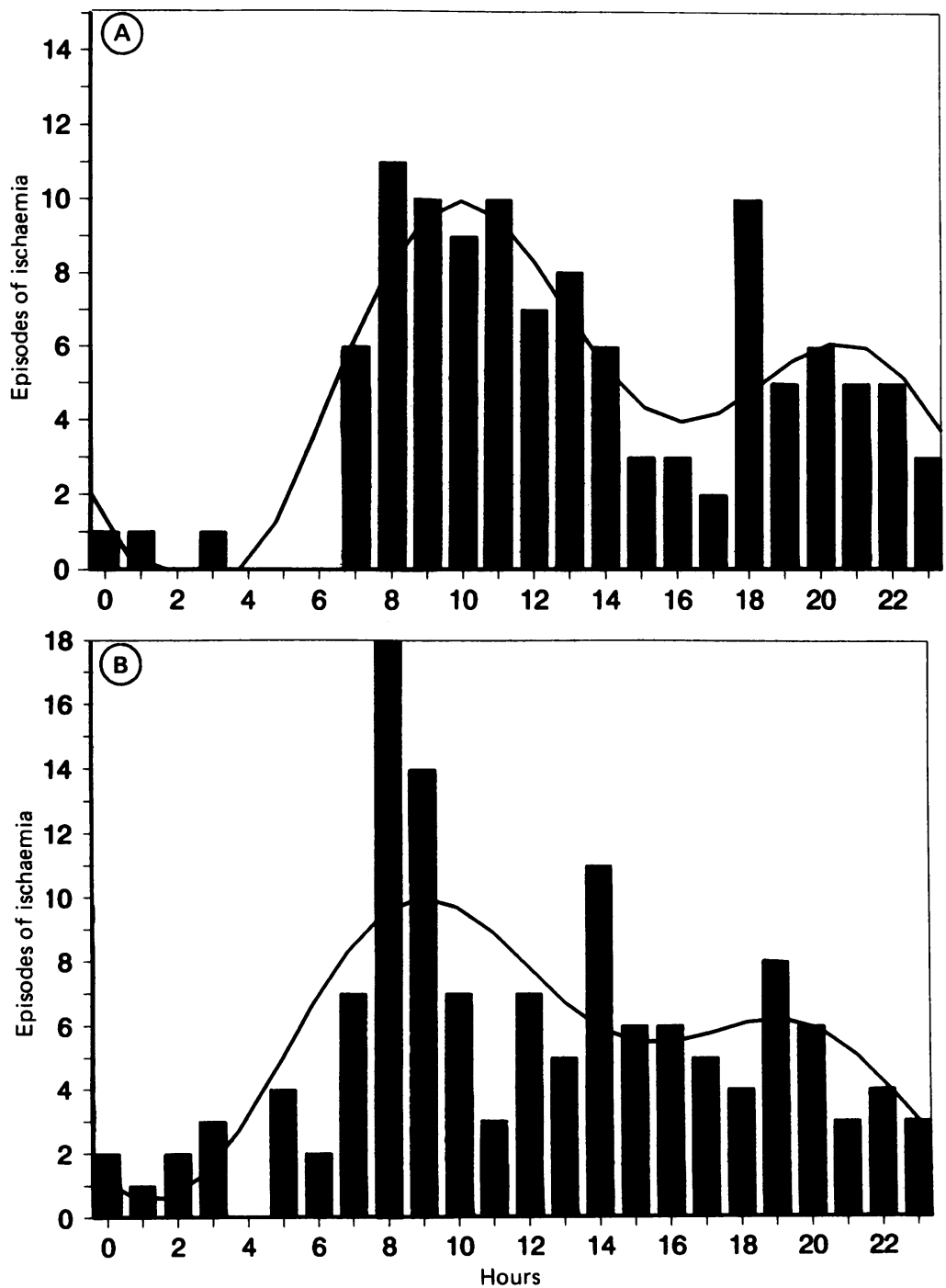

Figure 2 Twenty four hour circadian distribution of ischaemic episodes during placebo (A) and continuous transdermal nitrate treatment $(B)$. patients $(4 \cdot 7 \%)$ withdrew because of local rashes (three active, one placebo). In two patients angina became worse on active intermittent treatment. One patient collapsed suddenly and died during the day while receiving active intermittent treatment. In addition, one patient on continuous treatment had so much difficulty with shift work patterns that he withdrew from the study.

\section{Discussion}

The results of this study support the conclusion that transdermal glyceryl trinitrate significantly increases the total duration of treadmill exercise, the time to onset of angina, and the heart rate at $1 \mathrm{~mm} \mathrm{ST} \mathrm{segment} \mathrm{depression}$ when these variables were measured 3-5 hours after dosing and after two weeks' treatment when individually titrated doses were used intermittently. A significantly lower systolic blood pressure beneficially affected the ratepressure product. In contrast, the continuous treatment regimen of $10 \mathrm{mg} / 24 \mathrm{~h}$ was ineffective. It is not possible to conclude whether the higher doses used in the intermittent treatment group, the dosing interval, or a combination of the two helped to avoid the development of tolerance.

Tolerance to the hypotension and headache produced by organic nitrates has been known for over 80 years and is well accepted, but the magnitude and clinical relevance of tolerance to the anti-ischaemic and antianginal effects of organic nitrates have generated much controversy. There is evidence of a predictable improvement when organic nitrates are given three or four times a day during long term treatment, ${ }^{15} 16$ and an anti-ischaemic effect has been claimed in patients who applied transdermal glyceryl trinitrate patches daily during chronic treatment. ${ }^{17}$ Other studies, however, report significant tolerance after long term use of oral long acting nitrates ${ }^{1819}$ and transdermal patches. ${ }^{2021}$ Also, Moreyra and Kostis showed that a glyceryl trinitrate patch applied a few hours before coronary arteriography resulted in significant coronary artery dilatation, but when the patch was applied 24 hours beforehand this effect was strongly attenuated. ${ }^{22}$

This study accords with earlier studies which show that transdermal nitrates are effective when given intermittently, ${ }^{8}$ though interestingly Waters et al were unable to show a similar benefit on exercise testing of using intermittent transdermal glyceryl trinitrate; in most patients the side effects outweighed any demonstrable benefit. ${ }^{23}$ Recently in a large multicentre study of 206 patients a nitrate free 

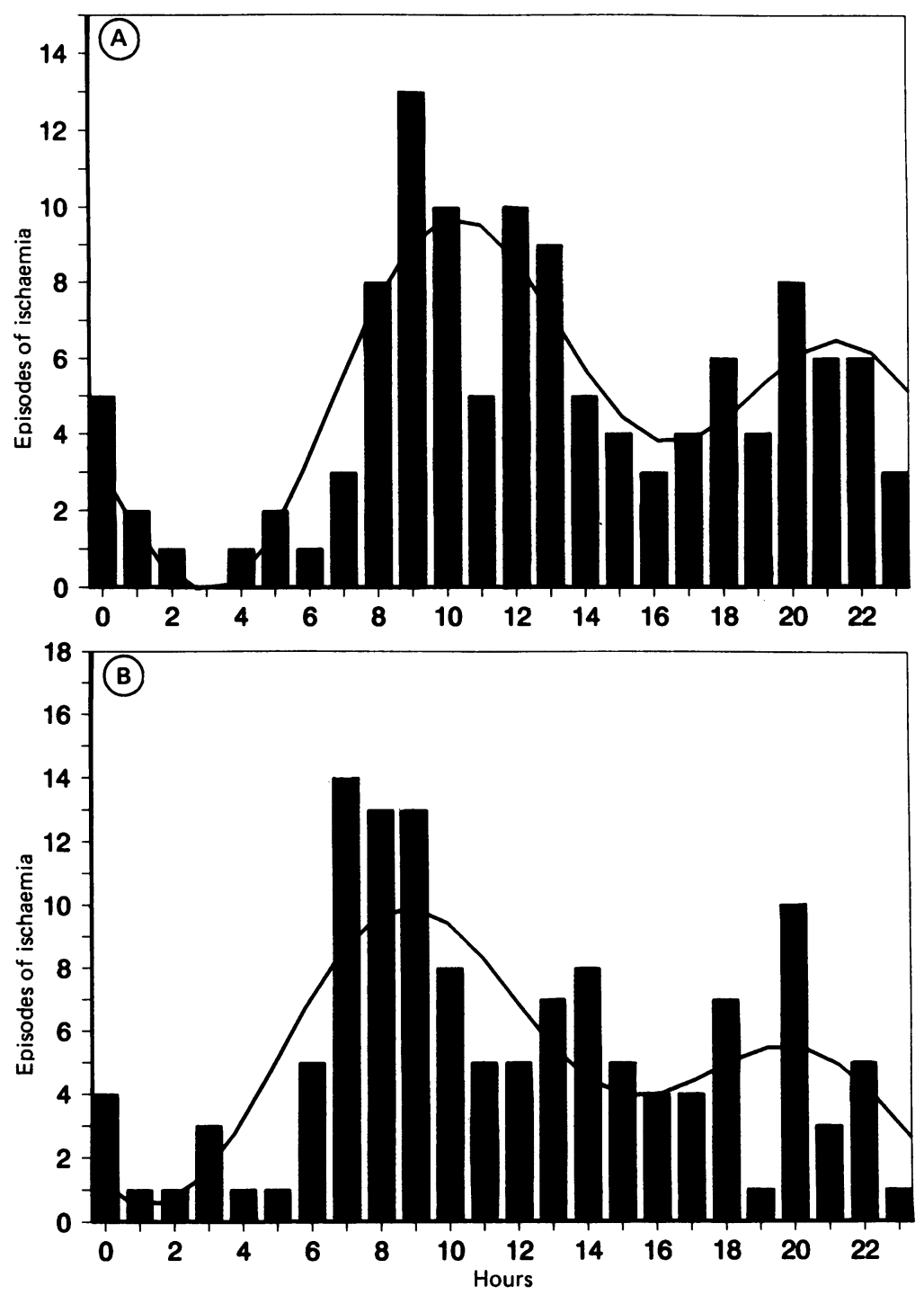

Figure 3 Twenty four hour circadian distribution of ischaemic episodes during placebo (A) and intermittent transdermal nitrate treatment $(B)$.

period of 12 hours seemed to prevent the development of tolerance, and prolongation of exercise duration was seen during the daytime. ${ }^{24}$ In this study, however, there were nine patients who fulfilled the defined criteria for a significant increase in rest angina during the nitrate-free periods during active intermittent treatment; in addition, the results for exercise testing before the reapplication of the patch the following morning were less good than with placebo. This has raised the question that "rebound" may be a real if rare phenomenon in patients treated with transdermal glyceryl trinitrate as monotherapy. In our study we investigated the circadian distribution of total and silent ischaemic episodes. We found that transdermal glyceryl trinitrate given intermittently or continuously did not alter the circadian distribution of ischaemic episodes and in particular that intermittent treatment was not associated with an increase in episodes during the night time hours. Clearly this does not rule out rebound in isolated cases but it does suggest that it must be rare if it occurs at all.

We thank Ciba-Geigy Pharmaceuticals for their support for the study, and in particular $\operatorname{Dr} M \mathrm{~A}$ Ford who helped set up and coordinate the trial.

1 Brunton TL. Use of nitrite of amyl in angina pectoris. Lancet 1867;ii:97-8.

2 Charash B, Scheidt SS. The controversy over transdermal nitroglycerin: an update. Am Heart $J$ 1986;112:215-7.

3 Abrahams $\mathrm{J}$. The brief saga of transdermal nitroglycerin discs: paradise lost? Am J Cardiol 1984;54:220-4.

4 Zeller FP, Klamerus K. Controversies in the use of transdermal nitroglycerin systems. Clin Pharmacol 1987;6: 605-16.

5 Cerri B, Grasso F, Cefis M, et al. Comparative evaluation of the effect of two doses of Nitroderm TTS on exercise related parameters in patients with angina pectoris. Eur Heart $J 1984 ; 5: 710-5$.

6 Colombo G, Favini G, Aglieri S, et al. Nitroderm TTS in exercise induced angina pectoris-a randomised double blind study. Int J Clin Pharmacol 1985;23:211-4.

7 Crean PA, Ribeiro P, Crea F, et al. Failure of transdermal nitroglycerin to improve chronic stable angina: a randomized, placebo-controlled, double-blind, double cross domized, placebo-controlled, double-blind,

8 Cowan JC, Bourke JP, Reid DS, et al. Prevention of tolerance to nitroglycerin patches by overnight removal. tolerance to nitroglycerin patch

9 Parker JO, Fung HL, Ruggirello D, et al. Tolerance to isosorbide dinitrate: rate of development and reversal. Circulation 1983;68:1074-80.

10 Luke R, Sharpe N, Coxon R. Transdermal nitroglycerin in angina pectoris: efficacy of intermittent application. $\mathrm{J} \mathrm{Am}$ Coll Cardiol 1987;10:642-6.

11 Needleman P, Johnson EM Jr. Mechanism of tolerance development to organic nitrates. J Pharmacol Exp Ther 1973;184:7009-15.

12 Ingarro LJ, Lippton H, Edwards JC, et al. Mechanism of vascular smooth muscle relaxation by organic nitrates, nitrates nitroprusside and nitric oxide: evidence for the involvement of $S$-nitrosothiols as active intermediates. J Pharmacol Exp Ther 1981;218:739-49.

13 Allexsson KL, Anderson RG. Tolerance towards nitroglycerin, induced in vivo, is correlated to a reduced cGMP glycerin, induced in vivo, is correlated to a reduced cGMP response and an alteration

14 Quyyumi AA, Mockus L, Wright C, Fox KM. Morphology of ambulatory ST segment changes in patients with varying severity of coronary artery disease: investigation of the frequency of nocturnal ischaemia and coronary spasm. Br Heart J 1985;53:186-93.

15 Lee G, Mason DT, DeMaria AN. Effects of long term and administration of isosorbide dinitrate on the antianginal response to nitroglycerin: absence of cross-reference and self-tolerance shown by exercise testing. $\mathrm{Am} \mathrm{J} \mathrm{Cardiol}$ 1978;41:82-7.

16 Danahy DT, Aronow WS. Hemodynamics and antianginal effects of high dose oral isosorbide dinitrate after chronic use. Circulation 1977;56:205-12.

17 Muieson G, Agabita-Rosei E, Muiesan L, et al. A multicenter trial of transdermal nitroglycerin in exercise induced angina: individual antianginal response after induced angina: individual antianginal response aster

18 Thadani U, Fung HL, Darke AC. Oral isosorbide dinitrate in angina pectoris: comparison of duration of action and dose-response relation during acute and sustained therapy. Am J Cardiol 1982;49:411-7.

19 Silber S, Krause KH, Garner C, Thiesen K, Jahrmarker H. Anti-ischemic effects of an $80 \mathrm{mg}$ tablet of isosorbide dinitrate in sustained-release form before and after 2 weeks treatment with $80 \mathrm{mg}$ once daily or twice daily. $Z$ Kardiol 1983;72(suppl 3):211-7.

20 Reichek N, Priest C, Zimrin D, et al. Antianginal effects of nitroglycerin patches. Am J Cardiol 1984;54:1-7.

21 Thadani U, Hamilton SF, Olson E, et al. Transdermal nitroglycerin patches in angina pectoris. Ann Intern Med 1986;105:485-92.

22 Moreyra AE, Kostis JB. Effect of cutaneous nitroglycerin patches on coronary artery diameter: issues concerning patches on coronary artery diameter: issues concerning 428-33.

23 Waters DD, Juneau M, Gossald M, Choquette G, Brien M. Limited usefulness of intermittent nitroglycerin patches in stable angina. $J$ Am Coll Cardiol 1989;13:421-5.

24 DeMots H, Glasser SP. Intermittent transdermal nitroglycerin therapy in the treatment of chronic stable angina. glycerin therapy in the treatment of
J Am Coll Cardiol 1989;13:786-93. 